\title{
COMMUNITY-BASED PEER-LED INTERVENTION FOR HEALTHY AGEING AND EVALUATION OF THE 'HAPPY’ PROGRAM
}

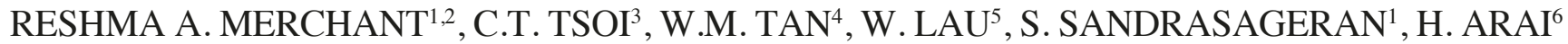

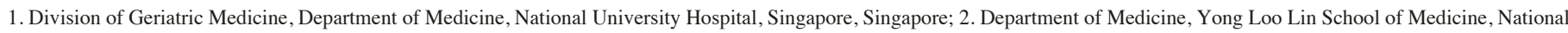
University of Singapore, Singapore; 3. Department of Psychological Medicine, National University Hospital, Singapore; 4. Integrated Health Division, MOH Office for Healthcare Transformation, Singapore; 5. Primary and Community Care Development Division, Agency for Integrated Care, Singapore; 6. National Center for Geriatrics and Gerontology, Obu, Aichi, Japan. Corresponding author: Associate Professor Reshma A Merchant, Division of Geriatric Medicine, Department of Medicine, National University Hospital, 1E Kent Ridge Road, Singapore 119228, Email: reshmaa@nuhs.edu.sg, ORCID iD: 0000-0002-9032-0184

\begin{abstract}
Objectives: Population ageing impacts many areas of society from health and social care cost to housing and future workforce, and whole-of-society approach is required to promote healthy ageing. The Decade of Healthy Ageing report has called upon multi-sectoral collaboration to promote age-friendly communities. The Healthy Ageing Promotion Program for You (HAPPY) is a community-based dual-task exercise program for older adults led by health coaches (HC) or trained volunteers (HAPPY leaders) to promote healthy ageing. The primary objective was to observe improvement in cognition. The secondary objective was to observe improvement in frailty status, functional status, perceived health and reduction of social isolation. We also aim to evaluate the effectiveness and describe the implementation of the HAPPY program. Design: To engage older adults with prefrailty, frailty and/or cognitive impairment in dual-task exercise program. Recruitment and publicity were through country-wide multisectoral collaboration. Setting: Community setting. Participants: More than 700 older adults participated in $\geq 50$ different sites including senior activity centres. Five hundred and sixtynine participants attended phase 1 screening. Pre-frail or frail ambulant participants or those with underlying cognitive impairment were invited to participate in phase 2 screening. Among them 296 participants enrolled in phase 2 with $66.6 \%$ follow up rate at 3 months. Measurements: Phase 1 and 2 screening consisted of screening for frailty (FRAIL), cognition (Montreal Cognitive Assessment [MoCA]), falls, vision, grip strength, perceived health (EuroQol vertical visual analogue scale), depression (geriatric depression scale), social network (6-item Lubben Social Network Scale), gait speed and physical function (Short physical performance battery [SPPB]). Results: HC led $61.7 \%$ of the participants, and HAPPY was conducted twice weekly for $64 \%$ of the participants. There was significant improvement in the MoCA scores both in the HC and HAPPY leaders' led groups. Overall physical function, chair-stand and balance domain improved significantly especially in the groups led by $\mathrm{HC}$ and those participating in twice-weekly exercises. There was significant improvement in perceived health, reduction in social isolation, improvement in frailty status and reduction of falls at 3 months. Conclusion: Community embedded peer-led program to promote healthy ageing like HAPPY can improve cognition, physical function, and frailty status, reduce social isolation, and improve perceived health. It takes a "village" to promote healthy ageing, and the need to have a life course approach to healthy longevity which must involve local government and ministerial organisations, non-profit organisations, industries, academia, and community to redesign health.
\end{abstract}

Key words: Healthy ageing, frailty, cognition, dual-task exercise, social isolation.

Number of older adults aged 65 years and over is projected to double to 1.5 billion by 2050 as a result of advancement in medical, public health, social and economic development leading to improvement in survival (1). The top three countries in Asia with the largest percentage point increase will be Republic of Korea, Singapore, and Taiwan Province of China. While lifespan continues to increase, healthspan has been slow to improve, and a person is known to spend the last decade of his or her life in poor health (2). The World Report on Ageing and Health by World Health Organisation (WHO) defines healthy ageing as the process of developing and maintaining functional ability that enables wellbeing. The interaction between individual and environment is crucial to achieve the optimum trajectory which can be modified to maintain a person's functional ability and intrinsic capacity throughout the life course. The United Nations General Assembly has just declared 2021-2030 as the Decade of Healthy Ageing where all stakeholders including government, private sectors, Received January 1, 2021 community and academia will need to come together to add life to years, with one of the areas of action include "ensure that communities foster the abilities of older people" through designing national / subnational programmes on age-friendly cities and communities (3).

Ageing in place, increasing physical activity, reducing loneliness, and maintaining functional ability including cognition amongst older people in fast aging countries is a public health priority. Frailty and dementia, both linked with ageing and chronic diseases are a major cause of disability and associated with poor quality of life both for the older adult and caregiver. Frailty is a dynamic clinical state of decreased functional reserve which increases individual's vulnerability to external stressors ranging from robust, prefrailty, frailty to frailty with disability, and frailty is reversible (4). Frailty and dementia have a bidirectional relationship, closely associated and often co-exist in the last few years of life (5). Based on WHO Guidelines on Integrated Care for Older People (ICOPE), Published online February 16, 2021, http://10.1007/s12603-021-1606-6 


\section{THE JOURNAL OF NUTRITION, HEALTH \& AGING}

significant functional decline and care dependency can be delayed with upstream screening and interventions through community level interventions and realigning health systems $(6$, 7).

Every country has different demographic challenges and identifying the mechanisms to activate the community to participate in the intervention programs, driver of behaviour change and sustenance is crucial to adapt to other programs (8). The Healthy Ageing Promotion Program for You (HAPPY) program was started in 2017 to engage older adults with prefrailty, frailty and/or cognitive impairment in dualtask exercise in the community setting led by health coaches or trained volunteers with the aim of improving function, cognition and reducing social isolation amongst community dwelling older people in Singapore. The planning and initiation of the program was driven by academia in partnership with National University Health System (NUHS) Regional Health System (RHS), the Agency for Integrated Care (AIC) and the People's Association (PA) with shared accountability. Healthcare system in Singapore is divided into three Integrated RHS clusters. Each of the cluster is responsible for the population health of the selected region, and work closely with AIC and PA in bringing together care, enhancing capabilities of community including community care and primary care providers, and to anchor care in the community as a team (9).

The primary objective of the program was to observe improvement in cognition. The secondary objective was to observe improvement in frailty status, functional status, perceived health and reduction of social isolation. In addition, our aim was also to evaluate and compare the effectiveness of the dual task exercise led by health coaches and trained volunteers.

\section{Methods}

\section{Recruitment and publicity}

The HAPPY program was initially started in the Western region of Singapore in 2017, and eventually scaled up across whole of Singapore over two years (Figure 1). The initial publicity for recruitment was through the NUHS RHS for the western community partners, AIC for senior care centres and senior activity centre, and PA for community dwelling older adults in their relevant community development council (Figure 2). Participants also opted to join the program through word of mouth from peers. Participants were invited to participate in phase 1 screening program to screen for frailty, depression, cognition, falls, vision, grip strength and gait speed. If participants screened positive for dementia or depression, they would be referred to the NUHS Geriatric Psychiatry OutReach Assessment, Consultation and Enablement team for management and follow up. Those with history of falls, weight loss and vision impairment were given a referral letter to see their general practitioner. Only participants who were screened to be pre-frail or frail and ambulant or had underlying cognitive impairment were invited to participate in phase 2 screening on functional status, physical activity, and social network. Participants were followed up at 3 and 6 months.

Figure 1

Scaling up of the Healthy Ageing Promotion Program for You (HAPPY)

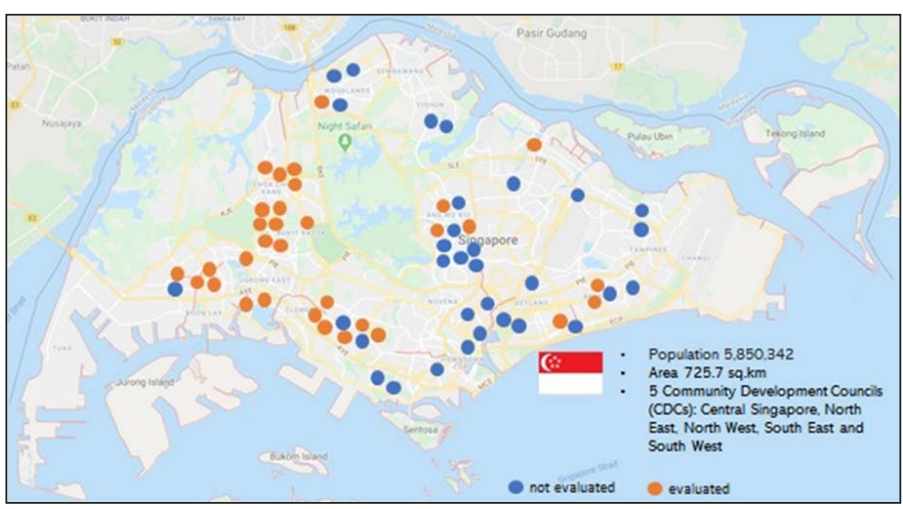

Figure 2

Recruitment and Publicity of the Healthy Ageing Promotion Program for You

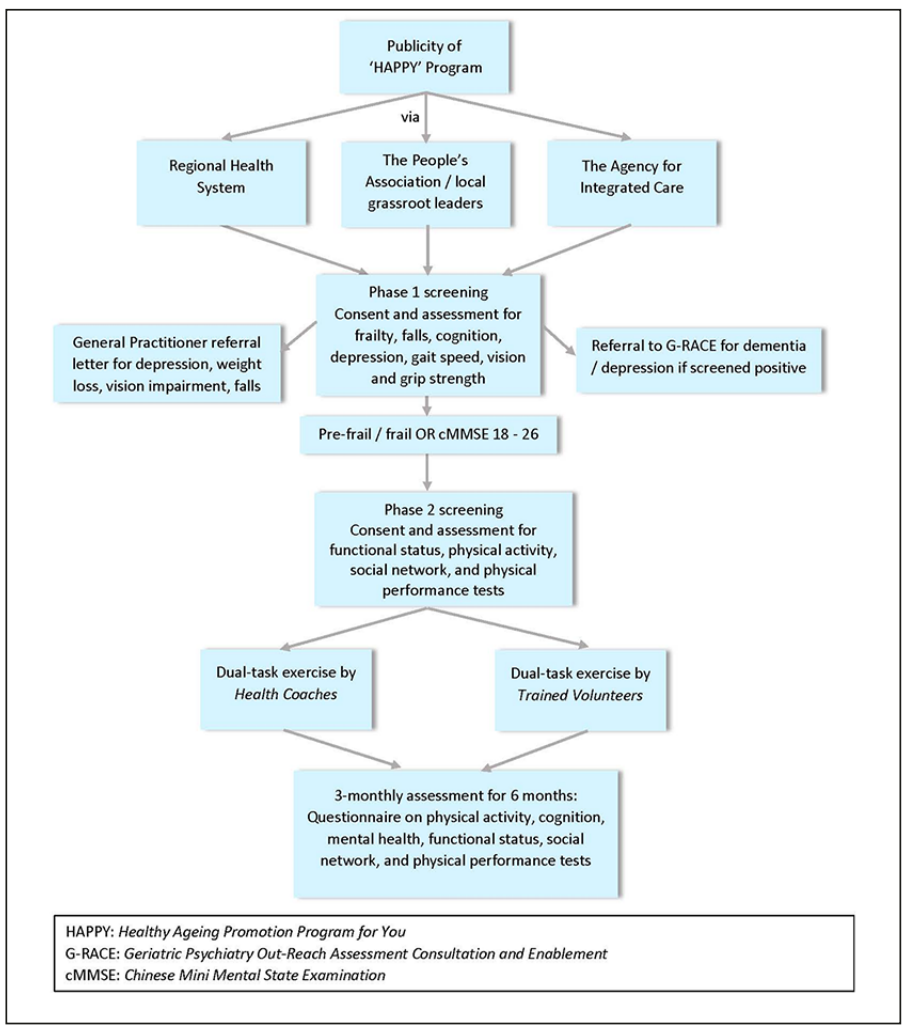

Although the inclusion criteria for evaluation follow-up on the dual-task exercise program was $\geq 60$ years old prefrail, frail and ambulant or cognitive impairment, there were no restrictions for those who were keen to participate but did not fulfill the inclusion criteria. There were more than 700 
Figure 3

Example of Healthy Ageing Promotion Program for You Manual and Guide

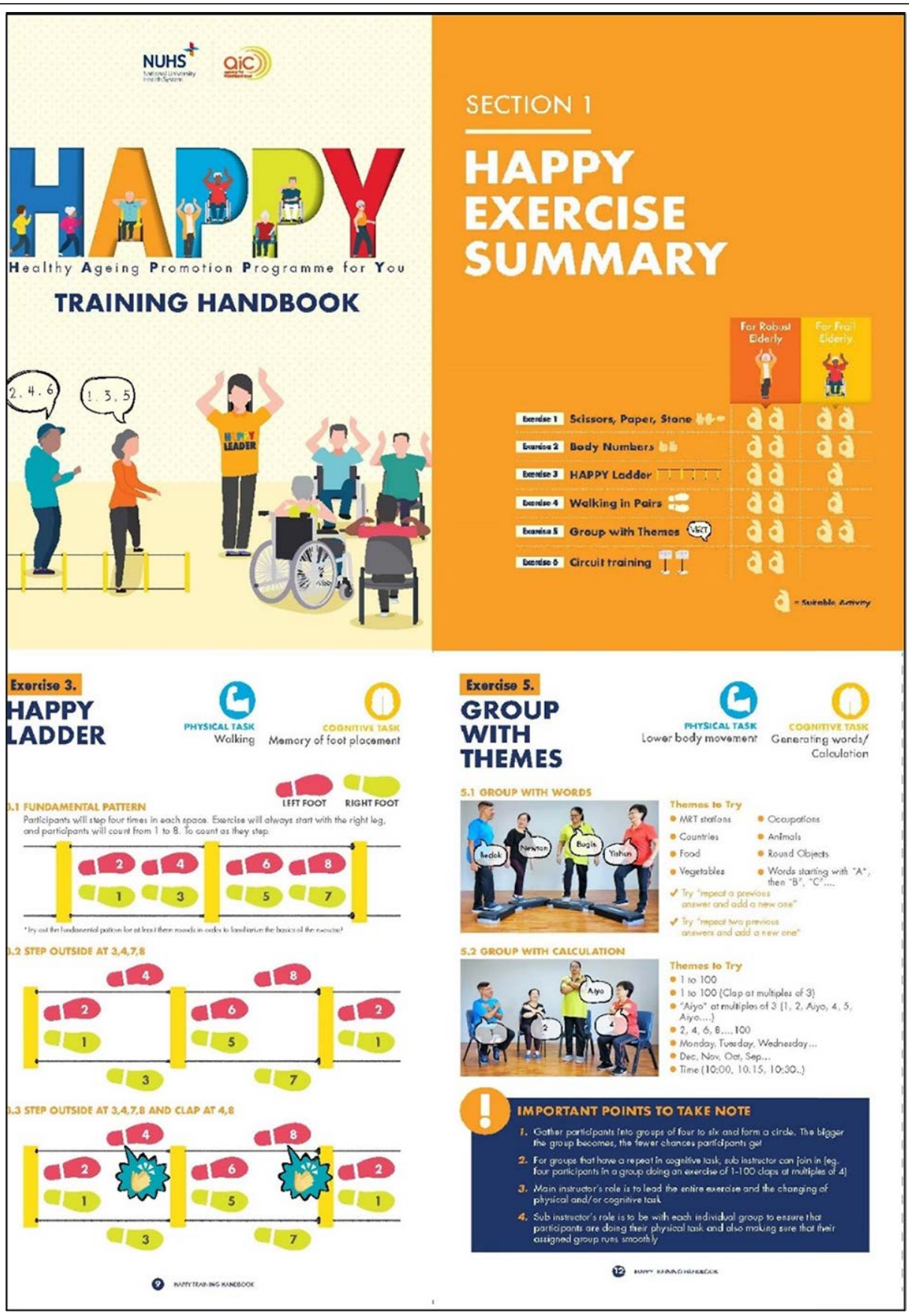

older adults participating in more than 50 sites island wide before Covid-19 restrictions were implemented in March 2020. Selected sites were chosen randomly for assessment and evaluation. Attendance was measured by the completeness of blood pressure monitoring prior to start of the exercise session. For phase 1 screening, 569 older adults were enrolled, and 296 attended Phase 2 screening. For those who consented, 33.4\% dropped out, and data was available for 197 participants at 3 months.
The evaluation is reported according to the Transparent Reporting of Evaluations with Nonrandomized Designs and description of the intervention from the Template for Intervention Description and Replication checklist $(10,11)$.

\section{Program Description}

HAPPY is a dual-task exercise program adapted from cognicise which originated from National Center for Geriatrics and Gerontology in Nagoya, Japan. Cognicise is a 
Figure 4

Volunteer (HAPPY leader) Training

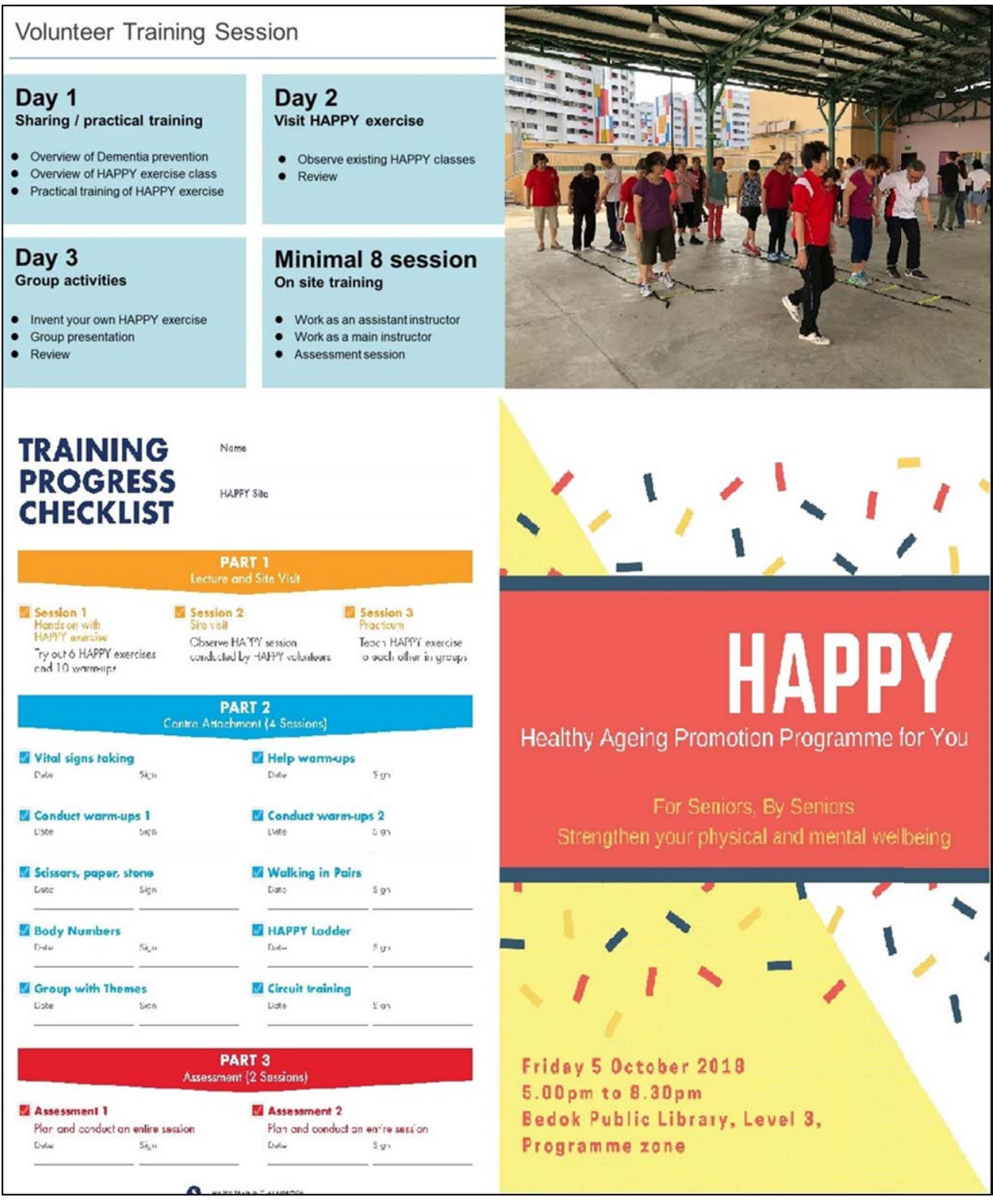

multicomponent exercise incorporating physical, cognitive, and social activities with the aim of improving cognition in older adults with mild cognitive impairment (12). These exercises had to be adapted locally as Singapore is a multiracial and multicultural country with Chinese, Indian and Malays being the three main ethnic groups, and varied education level. In addition, the exercise components include low to moderate intensity circuit and resistance training as secondary outcomes focussed on physical function. There are more than 80 different dual task combination of varying intensities co-created by the health coaches, volunteers, and participants. The examples of the dual task exercises are seen in the exercise manual which is given to volunteers (Figure 3) and includes scissors paper stone, body numbers, HAPPY ladder, walking in pairs, group with themes and circuit training. The exercises are conducted for 60 minutes once or twice weekly depending on the site and centre preference and availability of volunteers, and either led by health coaches or volunteers. The dual task components comprised 40 minutes of the total exercise program.

\section{Recruitment and Training of volunteers (HAPPY leaders)}

Happy leaders were identified either from the initial groups led by health coaches, community centres, senior care centres or the National Library Board volunteers. To date 625 certified HAPPY leaders have undergone structured training. The training includes theoretical aspect on dementia prevention, 
blood pressure and heart rate monitoring, evidence behind dual-task exercise, co-creation of new dual-task combination exercises, co-leading the exercises with health coaches and eventually leading the group independently. The HAPPY leaders need to lead or co-lead at least 8 sessions with the health coaches and must clear the assessments to be certified (Figure 4). The health coaches will conduct periodic on-site quality checks and obtain feedback from the participants to ensure the program is delivering its original objectives. Since there are only four trained health coaches, HAPPY leaders are the key drivers to ensure sustainability and scalability of the program. There are periodic celebrations to recognise and acknowledge HAPPY leaders.

\section{Outcome Measures}

The primary outcome was improvement in cognition, and secondary outcomes being frailty, physical function, social network, falls, depression and perceived health at 3 months.

\section{Primary outcomes}

Cognition was measured using the Montreal Cognitive Assessment (MoCA) score (13).

\section{Secondary outcomes}

Frailty was measured using the 5-item FRAIL scale (Fatigue, Resistance, Aerobic, Illness, and Loss of Weight) which has been validated locally (14). The scores range from 0 (robust), 1-2 (prefrail) and 3-5 (frail) (15). Geriatric depression scale was used to assess for depression, with a maximum score of 15 where $\geq 5$ is positive for depression (16). Social network was assessed using the 6-item Lubben Social Network Scale (LSNS-6). LSNS-6 is a brief, validated tool and includes size, closeness, and frequency of contact with friends and family members. The maximum total score is 30 and a score below 12 suggests that an individual is at risk of social isolation (17). Short physical performance battery (SPPB) test was used to measure physical function which includes balance, gait speed over 4 meters and five continuous chair-stand with a maximum score of 12. Perceived general health was assessed using EuroQol vertical visual analogue scale, ranging from "worst possible" to "best possible" health (18).

\section{Statistical Analysis}

All analysis was conducted using IBM SPSS Version 25. Differences between baseline and 3rd month follow up were calculated to derive positive values to indicate improvement. Significant differences between baseline and 3rd month follow up assessment were determined using paired sample t-tests. Differences in change between groups (health coach led and volunteer led) was determined using general linear model adjusted for age and baseline value. Statistical significance was set at $\mathrm{P}<0.05$.

\section{Ethics}

The study protocol including screening and intervention was approved by the National Healthcare Group (NHG) Domain Specific Review Board (DSRB). All participants signed an informed consent form.

\section{Results}

Health coach led $61.7 \%$ of the participants, and HAPPY leaders $38.3 \%$. HAPPY was conducted once weekly for $36.0 \%$, and twice weekly for $64.0 \%$ of the participants. There was significant improvement in the MoCA scores at 3 months in the entire group including health coach and HAPPY leaders' groups (Figure 5a). The improvement was more prevalent in the groups led by health coaches compared with the HAPPY leaders. The groups led by HAPPY leaders had overall lower baseline mean MoCA scores of 22.7 compared with 23.7 for the groups led by health coaches. For the SPPB scores, there was overall significant improvement from 9.7 to 10.3 , although improvement was most significant in the groups led by health coaches increasing from 9.6 to 10.4 and twice weekly exercises. There was significant improvement in the SPPB domains of balance and chair-stand for the entire group, groups led by health coaches and twice weekly exercises. There was nonsignificant improvement in gait speed for the entire group.

\section{Figure 5a}

Changes at 3rd month follow up for primary and secondary outcomes

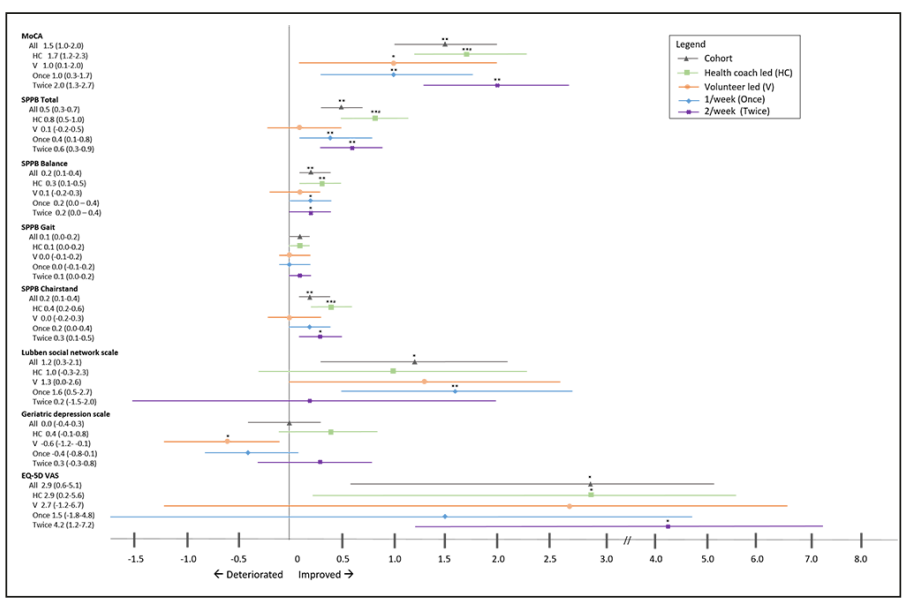

There was significant improvement in LSNS-6 scores with reduction of social isolation by $10 \%$ at 3 months for the entire group and those attending once weekly exercises. There was also significant improvement in the perceived health at 3 months in the entire group, groups lead by health coaches and those attending twice weekly exercises. There was no significant difference in depression prevalence which was generally low at $9.3 \%$, and the groups led by HAPPY leaders had a small significant increase in the GDS scores at 3 months. 


\section{THE JOURNAL OF NUTRITION, HEALTH \& AGING}

However, the prevalence of at least moderate to extreme depression or anxiety on the Euroqol-5D domain reduced by $30 \%$.

There was a significant increase in the number of robust older adults and a reduction in mean FRAIL scores in the entire group, groups led by health coaches and HAPPY leaders at 3 months. The prevalence of frailty reduced by $75 \%$ and $50 \%$ in the groups who attended twice weekly exercises and led by HAPPY leaders, respectively (Figure 5b). The prevalence of 1 or more falls reduced significantly by two thirds at 3 months from $29.1 \%$ to $10.2 \%$.

Figure 5b

Prevalence of frailty at baseline and 3rd month

\begin{tabular}{|c|c|c|c|c|c|c|c|c|c|c|}
\hline \multicolumn{11}{|c|}{ Frailty status at baseline and 3rd month } \\
\hline \multicolumn{3}{|c|}{ Mean Frail score } & & & & & & & & \\
\hline $100.0 \%$ & 0.86 & $0.43^{* *}$ & 0.81 & $0.44^{* *}$ & 0.91 & $0.41^{* *}$ & 0.85 & $0.47^{* *}$ & 0.86 & $0.34^{* *}$ \\
\hline $80.0 \%$ & & $28.6 \%$ & & $28.6 \%$ & & & & & & \\
\hline $60.0 \%$ & & & & & & & & & & \\
\hline $40.0 \%$ & & & & & & & & & & \\
\hline $20.0 \%$ & & & $3 \%$ & & & & & & & \\
\hline \multirow[t]{3}{*}{$0.0 \%$} & & & & & & & & & & \\
\hline & $0 \mathrm{~m}$ & $3 \mathrm{~m}$ & $0 \mathrm{~m}$ & $3 m$ & $0 \mathrm{~m}$ & $3 \mathrm{~m}$ & $0 \mathrm{~m}$ & $3 \mathrm{~m}$ & $0 \mathrm{~m}$ & $3 \mathrm{~m}$ \\
\hline & & & & & & & Once & week & & Neek \\
\hline & & & & D Robus & = Pre-1 & il $=\mathrm{Fr}$ & & & & \\
\hline
\end{tabular}

\section{Discussion}

Ageing of the population impacts many aspects of society including healthcare and social care cost, labour and financial market, housing, and long-term care facilities. Whole-of-society approach is required to promote healthy ageing and improve the life of older people, and community as a whole. The primary aim of the HAPPY program was to maintain and / or improve function and cognition amongst at risk older adults although many robust older adults opted to participate in the exercises. Embedding of the HAPPY program within the population was successful as there was multisectoral collaboration between academia, government, non-profit organisations, and community. Both the Decade of Health Ageing and The World report on ageing and health policy framework for healthy ageing have emphasized the need for multi-sectoral collaboration to ensure that integrated care and primary health services are accessible, person-centred care focussed on common goal of functional ability, and to have opportunity to age in an age-friendly environment including ongoing lifelong learning and ability to contribute to their communities while retaining autonomy and health $(3,19)$.

With the demographic shift, countries will see increasing numbers of older people with chronic conditions contributing to disability including sarcopenia, frailty, dementia, osteoporosis, and vision / hearing impairment. Social isolation, frailty, dementia, falls, and fractures are associated with increased morbidity and mortality. The prevalence of prefrailty in Singapore is $37 \%$, frailty $6 \%$ and half of frail older adults are still independent which is similar to data from nationally representative studies $(14,20)$. In the rural area in the United States, prevalence of frailty can be as high as $30.4 \%$ (21). Frailty is not synonymous with disability or comorbidities. Older adults define successful ageing as being able to maintain physical and cognitive ability whereas disease and productivity are much lower down the list in community surveys (22, 23 ). Frailty is potentially reversible through personalised multicomponent approach including physical exercise, management of polypharmacy, falls, nutrition, loneliness, cognitive impairment, and depression (4). Similarly, cognitive impairment is also amenable to interventions and demonstrate reversibility through multi-domain approach (24). There are emerging studies to suggest that even low level of physical activity is associated with reduced risk of all-cause mortality (25). Interventions incorporating simultaneous physical, cognitive, and social activities have been effective in improving cognitive function than either interventions alone $(12,26)$.

Most multicomponent exercise programs have been conducted in trial setting, and while it may be proven to be effective in randomized controlled trials or in observational studies, the translation into public health practice and system change including scalability and sustainability at the population level may still continue to be a challenge and may not produce the intended outcomes seen in trials (27). The HAPPY program is a form of system-based approach to enable successful ageing which was successfully implemented through multicollaborative approach to co-create sustainable and scalable interventions to achieve high level of physical and mental functioning, stronger social network, and better quality of life. Participants of the HAPPY program significantly improved in their functional ability including cognition, frailty status and physical function especially in the total SPPB scores, balance, and chair-stand domain. This correlated with reduction of falls by two thirds at 3 months. A recent systematic review has shown that interventions which included balance and functional exercise with a total weekly dose of $\geq 3$ hours can reduce falls by $42 \%$ (28). Another similar successful population level program in County Perry, Missouri involved screening older adults for geriatric syndrome using the rapid geriatric assessment with exercise and cognitive stimulation program which showed improvement in functional status (8).

Older persons, especially women who often outlive their spouse may be at risk of social isolation and loneliness, and financial insecurity. Almost 1 in 2 older adults are known to be at risk of social isolation in Singapore and worldwide(29). Participatory, productive engagement and group-based interventions designed to promote physical activity have been known to increase social connectedness, and reduce loneliness (30). Our community-based intervention program did reduce social isolation by $10 \%$ at 3 months with significant increases 
in LSNS-6 scores. Interestingly, improvement was greatest amongst the participants attending once weekly exercises. One possible explanation may include that they may be busy engaging with friends and family's at other times.

Retirees who are retired from workforce may spend more time in "roleless role" where they can contribute to the society in the form of volunteering. Volunteerism in the HAPPY program is a good example of "productive ageing" and "meaningful engagement" contributing to the betterment of senior communities through "do-it-together" approach. The proportion of older adults who volunteer in the US have increased by $65 \%$ since 1974 , and older adults are known to commit twice the time to volunteer activities (31). There are multiple benefits of volunteering including better physical health, better chronic disease control and functional status, lower mortality, better cognitive and mental health, improved perceived health and better social network (32). This was corroborated by HAPPY leaders where many saw improvement in their chronic disease control (33). Our program showed that structured training and assessment of the volunteers with periodic quality monitoring did produce similar cognitive outcomes to the health coaches conducting the exercises. The SPPB scores including the balance, chair-stand and gait domain scores for the groups led by health coaches showed greater improvement compared to HAPPY leaders, and possible reasons for this may be gaps in training on physical function aspect. The training was focused mainly on improving cognition. In addition, there was no specific training provided on exercises for different muscle groups, mitigating falls risk and theoretical aspect of physical exercise besides the stretching and cooling down exercises.

The public health impact of the HAPPY program has been described according to RE-AIM framework covering effectiveness, reach, adoption, implementation, and maintenance (34). The success of the HAPPY program is attributed to the concerted shared accountability and sustained collaboration between different stakeholders to co-create, co-own the initiative, and the role of academia in providing directions based on available evidence and evaluation. Certified HAPPY leaders were acknowledged, and their efforts celebrated in different platforms to ensure sustainability and scalability of the program. However, more can be done in regularly upskilling the HAPPY leaders in different aspects such as falls prevention, frailty screening, detecting red flags amongst older adults and incentivizing them with vouchers or similar to keep them motivated. There needs to be an agile, inspirational, and innovative leadership and team to drive the program on the ground.

Implementing country wide healthy ageing initiatives has its own challenges. One of the biggest challenges in clinical trial or observational studies is drop-outs. At 3 months, the drop-out rate was $33.4 \% \%$, and 6 months $49.0 \%$. However, there were many new participants who continued to join and eventually joined as HAPPY leaders. There was no follow-up on the dropouts to see if they had a medical reason, life event or other reasons related to the dual task exercise itself. Language barrier in a multiracial country like Singapore can be a barrier to group activities, and participants did drop out when they could not understand the instructions. Like many other communitybased programs, $75 \%$ of the participants were females. Future evaluations should include male preferences and motivational factors. Continuing to motivate HAPPY leaders also remained a challenge. In addition to motivation, health coaches had to ensure new dual-task exercises being introduced. Incentivizing them may keep them motivated and sustain behavioral change. Of the 625 trained HAPPY leaders, $85 \%$ continue to lead or co-lead the groups. While the program did reduce overall social isolation, only a few of the groups felt responsible for team members' wellness and had longing for togetherness or "kampung spirit".

During Covid-19 pandemic physical distancing requirement, the HAPPY program was converted to virtual and only $40 \%$ of the participants surveyed were digital ready and remained socially connected (35). Community-based program can only be made possible with fixed groupings to allow for better track and trace ability once restrictions are lifted post Covid-19 pandemic. In the current state, HAPPY programs in the senior care and senior activity centers are ongoing with limited number of participants, safe distancing, and strict infection control practice in place.

We do not have the profile of volunteers, but it is known that older adults are more likely to volunteer if they are married and have a spouse who also volunteers, work part time and highly educated. With reinforcement of retirement age in certain countries, greater volunteering opportunities and benefits, and the fact that people are living longer post-retirement, policymakers should provide incentives and promote paid "productive ageing".

Healthy ageing initiatives within the community and the ability to age well with dignity and purpose can be made a reality, but commitment is required from multiple sectors with joint accountability. Evidence suggests that even nonhealthcare professionals can be trained to screen for frailty, decline in intrinsic capacity and cognitive impairment, and implement necessary interventions. The WHO Integrated Care for Older People, ICOPE handbook Guidance and Rapid Geriatric Assessment which are both available in digital form are examples of person-centred assessment and management pathway that can be administered in primary care or community to prevent and /or enable early detection of age-related diseases $(36,37)$. Community re-design for healthy ageing should provide integrated social and healthcare and improve social connectivity, access to lifelong learning, financial security, and opportunity for "productive ageing". 


\section{THE JOURNAL OF NUTRITION, HEALTH \& AGING}

\section{Conclusion}

Community embedded program like HAPPY can improve cognition, functional status, reduce social isolation, reverse frailty, and improve perceived health. Similar improvements were also shown with volunteer led groups but to a smaller extend. Our program has highlighted that it takes a "village" to promote healthy ageing, and the need to have a life course approach to healthy longevity that must involve local government and ministerial organisations, non-profit organisations, industries, academia, and community to redesign health. Healthy and active older adults can be a resource to the community and provide economic and social benefits.

Conflict of interest: The authors declare no conflicts of interest for this work.

\section{References}

1. United Nations: World Population Ageing 2019

2. Crimmins EM. Lifespan and Healthspan: Past, Present, and Promise. The Gerontologist. 2015;55(6):901-11.

3. Decade Of Healthy Ageing 2020-2030. World Health Organisation. 2020

4. Dent E, Morley JE, Cruz-Jentoft AJ, Woodhouse L, Rodriguez-Manas L, Fried LP, et al. Physical Frailty: ICFSR International Clinical Practice Guidelines for Identification and Management. J Nutr Health Aging. 2019;23(9):771-87.

5. Cano C, Samper-Ternent R, Al Snih S, Markides K, Ottenbacher KJ. Frailty and cognitive impairment as predictors of mortality in older Mexican Americans. J Nutr Health Aging. 2012;16(2):142-7.

6. Thiyagarajan JA, Araujo de Carvalho I, Pena-Rosas JP, Chadha S, Mariotti SP, Dua $\mathrm{T}$, et al. Redesigning care for older people to preserve physical and mental capacity: WHO guidelines on community-level interventions in integrated care. PLoS Med. 2019;16(10):e1002948.

7. WHO. Integrated care for older people: guidelines on community-level interventions to manage declines in intrinsic capacity. Geneva: World Health Organization; 2017.

8. Lundy J, Hayden D, Pyland S, Berg-Weger M, Malmstrom TK, Morley JE. An AgeFriendly Health System. J Am Geriatr Soc. 2020.

9. Merchant RA. COVID-19: Role of Integrated Regional Health System Towards Controlling Pandemic in the Community, Intermediate and Long-Term Care. The Journal of Frailty \& Aging. 2020;9(4):248-9.

10. Hoffmann TC, Glasziou PP, Boutron I, Milne R, Perera R, Moher D, et al. Better reporting of interventions: template for intervention description and replication (TIDieR) checklist and guide. BMJ. 2014;348:g1687.

11. Des Jarlais DC, Lyles C, Crepaz N, Group T. Improving the reporting quality of nonrandomized evaluations of behavioral and public health interventions: the TREND statement. Am J Public Health. 2004;94(3):361-6.

12. Shimada H, Makizako H, Doi T, Park H, Tsutsumimoto K, Verghese J, et al. Effects of Combined Physical and Cognitive Exercises on Cognition and Mobility in Patients With Mild Cognitive Impairment: A Randomized Clinical Trial. J Am Med Dir Assoc. 2018;19(7):584-91.

13. Dong Y, Lee WY, Basri NA, Collinson SL, Merchant RA, Venketasubramanian $\mathrm{N}$, et al. The Montreal Cognitive Assessment is superior to the Mini-Mental State Examination in detecting patients at higher risk of dementia. Int Psychogeriatr. 2012;24(11):1749-55

14. Merchant RA, Chen MZ, Tan LWL, Lim MY, Ho HK, van Dam RM. Singapore Healthy Older People Everyday (HOPE) Study: Prevalence of Frailty and Associated Factors in Older Adults. J Am Med Dir Assoc. 2017.

15. Malmstrom TK, Miller DK, Morley JE. A comparison of four frailty models. J Am Geriatr Soc. 2014;62(4):721-6.

16. D'Ath P, Katona P, Mullan E, Evans S, Katona C. Screening, detection and management of depression in elderly primary care attenders. I: The acceptability and performance of the 15 item Geriatric Depression Scale (GDS15) and the development of short versions. Fam Pract. 1994;11(3):260-6.
17. Lubben J, Blozik E, Gillmann G, Iliffe S, von Renteln Kruse W, Beck JC, et al. Performance of an abbreviated version of the Lubben Social Network Scale among three European community-dwelling older adult populations. Gerontologist. 2006;46(4):503-13

18. Brooks R. EuroQol: the current state of play. Health Policy. 1996;37(1):53-72

19. Beard JR, Officer A, de Carvalho IA, Sadana R, Pot AM, Michel JP, et al. The World report on ageing and health: a policy framework for healthy ageing. Lancet. 2016;387(10033):2145-54.

20. O'Caoimh R, Sezgin D, O'Donovan MR, Molloy DW, Clegg A, Rockwood K, et al. Prevalence of frailty in 62 countries across the world: a systematic review and metaanalysis of population-level studies. Age Ageing. 2020.

21. Sanford AM, Morley JE, Berg-Weger M, Lundy J, Little MO, Leonard K, et al. High prevalence of geriatric syndromes in older adults. PLoS One. 2020;15(6):e0233857.

22. Reich AJ, Claunch KD, Verdeja MA, Dungan MT, Anderson S, Clayton CK, et al What Does "Successful Aging" Mean to you? - Systematic Review and Cross-Cultural Comparison of Lay Perspectives of Older Adults in 13 Countries, 2010-2020. J Cross Cult Gerontol. 2020;35(4):455-78.

23. Whitley E, Benzeval M, Popham F. Population Priorities for Successful Aging: A Randomized Vignette Experiment. J Gerontol B Psychol Sci Soc Sci. 2020;75(2):293302

24. Ngandu T, Lehtisalo J, Solomon A, Levalahti E, Ahtiluoto S, Antikainen R, et al. A 2 year multidomain intervention of diet, exercise, cognitive training, and vascular risk monitoring versus control to prevent cognitive decline in at-risk elderly people (FINGER): a randomised controlled trial. Lancet. 2015;385(9984):2255-63.

25. Ramakrishnan R, He J-R, Ponsonby A-L, Woodward M, Rahimi K, Blair SN, et al Objectively measured physical activity and all cause mortality: A systematic review and meta-analysis. Preventive Medicine. 2020:106356.

26. Oswald WD, Gunzelmann T, Rupprecht R, Hagen B. Differential effects of single versus combined cognitive and physical training with older adults: the SimA study in a 5-year perspective. Eur J Ageing. 2006;3(4):179.

27. Casas-Herrero A, Anton-Rodrigo I, Zambom-Ferraresi F, Sáez de Asteasu ML, Martinez-Velilla N, Elexpuru-Estomba J, et al. Effect of a multicomponent exercise programme (VIVIFRAIL) on functional capacity in frail community elders with cognitive decline: study protocol for a randomized multicentre control trial. Trials. 2019;20(1):362.

28. Sherrington C, Fairhall N, Kwok W, Wallbank G, Tiedemann A, Michaleff ZA, et al. Evidence on physical activity and falls prevention for people aged $65+$ years: systematic review to inform the WHO guidelines on physical activity and sedentary behaviour. Int J Behav Nutr Phys Act. 2020;17(1):144.

29. Merchant RA, Liu SG, Lim JY, Fu X, Chan YH. Factors associated with social isolation in community-dwelling older adults: a cross-sectional study. Qual Life Res. 2020;29(9):2375-81.

30. Mays AM, Kim S, Rosales K, Au T, Rosen S. The Leveraging Exercise to Age in Place (LEAP) Study: Engaging Older Adults in Community-Based Exercise Classes to Impact Loneliness and Social Isolation. The American Journal of Geriatric Psychiatry. 2020 .

31. Gonzales E, Matz-Costa C, Morrow-Howell N. Increasing Opportunities for the Productive Engagement of Older Adults: A Response to Population Aging. The Gerontologist. 2015;55(2):252-61.

32. Carr DC, Fried LP, Rowe JW. Productivity \& Engagement in an Aging America: The Role of Volunteerism. Daedalus. 2015;144(2):55-67.

33. Metabolic syndrome, which increases the risk of heart disease, affects one in two seniors here. The Straits Times. 2020.

34. Glasgow RE, Vogt TM, Boles SM. Evaluating the public health impact of health promotion interventions: the RE-AIM framework. American journal of public health 1999;89(9):1322-7

35. Merchant RA, Chen MZ, Ng SE, Sandrasageran S, Wong BLL. Letter to the Editor: The Role of a Geriatrician Has Become Even More Important in an Academic Institution during COVID-19. J Nutr Health Aging. 2020;24(6):681-2.

36. Takeda C, Guyonnet S, Sumi Y, Vellas B, Araujo de Carvalho I. Integrated Care for Older People and the Implementation in the INSPIRE Care Cohort. J Prev Alzheimers Dis. 2020;7(2):70-4.

37. Merchant RA, Hui RJY, Kwek SC, Sundram M, Tay A, Jayasundram J, et al. Rapid Geriatric Assessment Using Mobile App in Primary Care: Prevalence of Geriatric Syndromes and Review of Its Feasibility. Frontiers in Medicine. 2020;7(261) 